\title{
The Number of Nilpotent Semigroups of Degree 3
}

\author{
Andreas Distler* \\ Centro de Álgebra da Universidade de Lisboa \\ Av. Prof. Gama Pinto, 2 \\ 1649-003 Lisboa \\ Portugal \\ adistler@fc.ul.pt
}

\author{
J. D. Mitchell \\ University of St Andrews, \\ Mathematical Institute, \\ North Haugh, \\ St Andrews, \\ Fife, KY16 9SS, \\ Scotland, UK \\ jdm3@st-and.ac.uk
}

Submitted: Jan 16, 2012; Accepted: Jun 15, 2012; Published: Jun 28, 2012 Mathematics Subject Classifications: 05A15, 20M99

\begin{abstract}
A semigroup is nilpotent of degree 3 if it has a zero, every product of 3 elements equals the zero, and some product of 2 elements is non-zero. It is part of the folklore of semigroup theory that almost all finite semigroups are nilpotent of degree 3.

We give formulae for the number of nilpotent semigroups of degree 3 on a set with $n \in \mathbb{N}$ elements up to equality, isomorphism, and isomorphism or anti-isomorphism. Likewise, we give formulae for the number of nilpotent commutative semigroups on a set with $n$ elements up to equality and up to isomorphism.
\end{abstract}

Keywords: nilpotent semigroups; power group enumeration; nilpotency degree

\section{Introduction}

The topic of enumerating finite algebraic or combinatorial objects of a particular type is classical. Many theoretical enumeration results were obtained thanks to the advanced orbit counting methods developed by Redfield [Red27], Polya [Pol37], and de Bruijn [dB59]. Numerous applications of the method known as power group enumeration can be found in [HP73]. Of particular interest for this paper is the usage to count universal algebras in $[$ Har66].

The enumeration of finite semigroups has mainly been performed by exhaustive search and the results are therefore restricted to very small orders. The most recent numbers

\footnotetext{
${ }^{*}$ Supported by the University of St Andrews and the project PTDC/MAT/101993/2008 of Centro de Álgebra da Universidade de Lisboa, financed by FCT and FEDER.
} 
are of semigroups of order 9 [Dis10], of semigroups with identity of order 10 [DK09], commutative semigroups of order 10 [Gri03], and linearly ordered semigroups of order 7 [Sla95].

In this paper we use power group enumeration to develop formulae for the number of semigroups of a particular type, which we now define.

A semigroup $S$ is nilpotent if there exists a $r \in \mathbb{N}$ such that the set

$$
S^{r}=\left\{s_{1} s_{2} \cdots s_{r} \mid s_{1}, s_{2}, \ldots, s_{r} \in S\right\}
$$

has size 1. If $r$ is the least number such that $\left|S^{r}\right|=1$, then we say that $S$ has (nilpotency) degree $r$.

As usual, the number of 'structural types' of objects is of greater interest than the number of distinct objects. Let $S$ and $T$ be semigroups. Then a function $f: S \rightarrow T$ is an isomorphism if it is a bijection and $f(x \cdot y)=f(x) \cdot f(y)$ for all $x, y \in S$. The dual $S^{*}$ of $S$ is the semigroup with multiplication $*$ defined by $x * y=y \cdot x$ on the set $S$. A bijection $f: S \rightarrow T$ is an anti-isomorphism if $f$ is an isomorphism from $S^{*}$ to $T$. Throughout this article we distinguish between the number of distinct semigroups on a set, the number up to isomorphism, and the number up to isomorphism or anti-isomorphism. We shall refer to the number of distinct semigroups that can be defined on a set as the number up to equality.

For $n \in \mathbb{N}$ we let $z(n)$ denote the number of nilpotent semigroups of degree 3 on $\{1,2, \ldots, n\}$. The particular interest in nilpotent semigroups of degree 3 stems from the observation that almost all finite semigroups are of this type. More precisely, Kleitman, Rothschild, and Spencer identified $z(n)$ in [KRS76] as an asymptotic lower bound for the number of all semigroups on that set. Furthermore, Jürgensen, Migliorini, and Szép suspected in [JMS91] that $z(n) / 2 n$ ! was a good lower bound for the number of semigroups with $n$ elements up to isomorphism or anti-isomorphism based on the comparison of these two numbers for $n=1,2, \ldots, 7$. This belief was later supported by Satoh, Yama, and Tokizawa [SYT94, Section 8] and the first author [Dis10] in their analyses of the semigroups with orders 8 and 9 , respectively.

This paper is structured as follows: in the next section we present and discuss our results, delaying certain technical details for later sections; in Section 3 we describe a way to construct semigroups of degree 2 or 3; in Section 4 nilpotent semigroups of degree 3 are considered up to equality; in Section 5 we present the relevant background material from power group enumeration and a number of technical results in preparation for Section 6 where we give the proofs for our main theorems. Tables containing the first few terms of the sequences defined by the various formulae in the paper can be found at the appropriate points. The implementation used to obtain these numbers is provided as the function Nr3NilpotentSemigroups in the computer algebra system GAP [GAP08] by the package Smallsemi [DM11]. 


\section{Formulae for the number of nilpotent semigroups of degree 3}

\section{$2.1 \quad$ Up to equality}

The number of nilpotent and commutative nilpotent semigroups of degree 3 on a finite set can be computed using formulae given in [JMS91, Theorems 15.3 and 15.8]. We

summarise the relevant results in the following theorem. As the theorems in [JMS91] are stated incorrectly we shall give a proof for Theorem 1 in Section 4.

Theorem 1. For $n \in \mathbb{N}$ the following hold:

(i) the number of distinct nilpotent semigroups of degree 3 on $\{1,2, \ldots, n\}$ is

$$
\sum_{m=2}^{a(n)}\left(\begin{array}{c}
n \\
m
\end{array}\right) m \sum_{i=0}^{m-1}(-1)^{i}\left(\begin{array}{c}
m-1 \\
i
\end{array}\right)(m-i)^{\left((n-m)^{2}\right)}
$$

where $a(n)=\lfloor n+1 / 2-\sqrt{n-3 / 4}\rfloor ;$

(ii) the number of distinct commutative nilpotent semigroups of degree 3 on $\{1,2, \ldots, n\}$ is

$$
\begin{aligned}
& \qquad \sum_{m=2}^{c(n)}\left(\begin{array}{c}
n \\
m
\end{array}\right) m \sum_{i=0}^{m-1}(-1)^{i}\left(\begin{array}{c}
m-1 \\
i
\end{array}\right)(m-i)^{(n-m)(n-m+1) / 2} \\
& \text { where } c(n)=\lfloor n+3 / 2-\sqrt{2 n+1 / 4}\rfloor .
\end{aligned}
$$

Note that there are no nilpotent semigroups of degree 3 with fewer than 3 elements. Accordingly, the formulae in Theorem 1 yield that the number of nilpotent and commutative nilpotent semigroups of degree 3 with 1 or 2 elements is 0 . The first few non-zero terms of the sequences given by Theorem 1 are shown in Tables 1 and 2 .

\subsection{Up to isomorphism and up to isomorphism or anti-isomorphism}

Our main results are explicit formulae for the number of nilpotent and commutative nilpotent semigroups of degree 3 on any finite set up to isomorphism and up to isomorphism or anti-isomorphism. As every commutative semigroup is equal to its dual we obtain three different formulae.

If $j$ is a partition of $n \in \mathbb{N}$, written as $j \vdash n$, then we denote by $j_{i}$ the number of summands equalling $i$. The first of our main theorems, dealing with nilpotent semigroups of degree 3 up to isomorphism, can then be stated as follows: 
Table 1: Numbers of nilpotent semigroups of degree 3 up to equality

\begin{tabular}{rl}
\hline$n$ & number of nilpotent semigroups of degree 3 on $\{1,2, \ldots, n\}$ \\
\hline 3 & 6 \\
4 & 180 \\
5 & 11720 \\
6 & 3089250 \\
7 & 5944080072 \\
8 & 147348275209800 \\
9 & 38430603831264883632 \\
10 & 90116197775746464859791750 \\
11 & 2118031078806486819496589635743440 \\
12 & 966490887282837500134221233339527160717340 \\
13 & 17165261053166610940029331024343115375665769316911576 \\
14 & 6444206974822296283920298148689544172139277283018112679406098010 \\
\hline
\end{tabular}

Table 2: Numbers of commutative nilpotent semigroups of degree 3 up to equality

\begin{tabular}{rl}
\hline$n$ & number of commutative nilpotent semigroups of degree 3 on $\{1,2, \ldots, n\}$ \\
\hline 3 & 6 \\
5 & 1620 \\
6 & 67170 \\
7 & 7655424 \\
8 & 2762847752 \\
9 & 3177531099864 \\
10 & 11942816968513350 \\
11 & 170387990514807763280 \\
12 & 11445734473992302207677404 \\
13 & 3783741947416133941828688621484 \\
14 & 5515869594360617154295309604962217274 \\
15 & 33920023793863706955629537246610157737736800 \\
16 & 961315883918211839933605601923922425713635603848080 \\
17 & 160898868329022121111520489011089643697943356922368997915120 \\
\hline
\end{tabular}


Table 3: Numbers of nilpotent semigroups of degree 3 up to isomorphism

\begin{tabular}{rl}
\hline$n$ & number of non-isomorphic nilpotent semigroups of degree 3 of order $n$ \\
\hline 3 & 1 \\
4 & 9 \\
5 & 118 \\
6 & 4671 \\
7 & 1199989 \\
8 & 3661522792 \\
9 & 105931872028455 \\
10 & 24834563582168716305 \\
11 & 53061406576514239124327751 \\
12 & 2017720196187069550262596208732035 \\
13 & 2756576827989210680367439732667802738773384 \\
14 & 73919858836708511517426763179873538289329852786253510 \\
15 & 29599937964452484359589007277447538854227891149791717673581110642 \\
\hline
\end{tabular}

Theorem 2. Let $n, p, q \in \mathbb{N}$. For $1 \leqslant q<p$ denote

$$
N(p, q)=\sum_{j \vdash q-1} \sum_{k \vdash p-q}\left(\prod_{i=1}^{q-1} j_{i} ! i^{j_{i}} \prod_{i=1}^{p-q} k_{i} ! i^{k_{i}}\right)^{-1} \prod_{a, b=1}^{p-q}\left(1+\sum_{d \mid \operatorname{lcm}(a, b)} d j_{d}\right)^{k_{a} k_{b} \operatorname{gcd}(a, b)} .
$$

Then the number of nilpotent semigroups of degree 3 and order $n$ up to isomorphism equals

$$
\sum_{m=2}^{a(n)}(N(n, n)-N(n-1, m-1)) \text { where } a(n)=\lfloor n+1 / 2-\sqrt{n-3 / 4}\rfloor,
$$

The second of our main theorems gives the number of nilpotent semigroups of degree 3 up to isomorphism or anti-isomorphism.

Theorem 3. Let $n, p, q \in \mathbb{N}$. For $1 \leqslant q<p$ let $N(p, q)$ as in (1) and denote

$$
L(p, q)=\frac{1}{2} N(p, q)+\frac{1}{2} \sum_{j \vdash q-1} \sum_{k \vdash p-q}\left(\prod_{i=1}^{q-1} j_{i} ! i^{j_{i}} \prod_{i=1}^{p-q} k_{i} ! i^{k_{i}}\right)^{-1} \prod_{a=1}^{p-q}\left(q_{a}^{k_{a}} p_{a, a}^{k_{a}^{2}-k_{a}} \prod_{b=1}^{a-1} p_{a, b}^{2 k_{a} k_{b}}\right)
$$

where

$$
p_{a, b}=\left(1+\sum_{d \mid \operatorname{lcm}(2, a, b)} d j_{d}\right)^{a b / \operatorname{lcm}(2, a, b)}
$$

and

$$
q_{a}=\left\{\begin{array}{lll}
\left(1+\sum_{d \mid a} d j_{d}\right)\left(1+\sum_{d \mid 2 a} d j_{d}\right)^{(a-1) / 2} & \text { if } a \equiv 1 & \bmod 2 \\
\left(1+\sum_{d \mid a} d j_{d}\right)^{a} & \text { if } a \equiv 0 & \bmod 4 \\
\left(1+\sum_{d \mid a / 2} d j_{d}\right)^{2}\left(1+\sum_{d \mid a} d j_{d}\right)^{a-1} & \text { if } a \equiv 2 & \bmod 4
\end{array}\right.
$$


Table 4: Numbers of nilpotent semigroups of degree 3 up to isomorphism or antiisomorphism

\begin{tabular}{rl}
\hline$n$ & number of non-(anti-)isomorphic nilpotent semigroups of degree 3 of order $n$ \\
\hline 3 & 1 \\
4 & 8 \\
5 & 84 \\
6 & 2660 \\
7 & 609797 \\
8 & 1831687022 \\
9 & 52966239062973 \\
10 & 12417282095522918811 \\
11 & 26530703289252298687053072 \\
12 & 1008860098093547692911901804990610 \\
13 & 1378288413994605341053354105969660808031163 \\
14 & 36959929418354255758713676933402538920157765946956889 \\
15 & 14799968982226242179794503639146983952853044950740907666303436922 \\
\hline
\end{tabular}

Then the number of nilpotent semigroups of degree 3 and order $n$ up to isomorphism or anti-isomorphism equals

$$
\sum_{m=2}^{a(n)}(L(n, m)-L(n-1, m-1)) \quad \text { where } a(n)=\lfloor n+1 / 2-\sqrt{n-3 / 4}\rfloor .
$$

A semigroup is self-dual if it is isomorphic to its dual. The concept of anti-isomorphism has no relevance for self-dual semigroups. Combining Theorems 2 and 3, it is possible to deduce a formula for the number of self-dual, nilpotent semigroups of degree 3 up to isomorphism. More generally, considering semigroups of a certain type the number of self-dual semigroups up to isomorphism is equal to twice the number of semigroups up to isomorphism and anti-isomorphism minus the number of semigroups up to isomorphism.

Corollary 4. Let $n \in \mathbb{N}$ and let $N(p, q)$ and $L(p, q)$ be as defined in (1) and (2), respectively. Then the number of self-dual, nilpotent semigroups of degree 3 and order $n$ up to isomorphism equals

$$
\begin{aligned}
\sum_{m=2}^{a(n)}(2 L(n, m)-N(n, m)-2 L(n-1, m-1) & +N(n-1, m-1)) \\
& \text { where } a(n)=\lfloor n+1 / 2-\sqrt{n-3 / 4}\rfloor .
\end{aligned}
$$

Substituting in the previous corollary the actual formula for $2 L(p, q)$ we notice that $N(p, q) / 2$ appears as a term in $L(p, q)$ and cancels. The resulting simplified formula is implemented as part of the function Nr3NilpotentSemigroups in Smallsemi [DM11].

Since commutative semigroups are self-dual, we obtain just one formula up to isomorphism for commutative nilpotent semigroups of degree 3 . 
Table 5: Numbers of self-dual nilpotent semigroups of degree 3 up to isomorphism

\begin{tabular}{rl}
\hline$n$ & number of non-isomorphic self-dual nilpotent semigroups of degree 3 of order $n$ \\
\hline 3 & 1 \\
5 & 7 \\
6 & 649 \\
7 & 19605 \\
8 & 1851252 \\
9 & 606097491 \\
10 & 608877121317 \\
11 & 1990358249778393 \\
12 & 25835561207401249185 \\
13 & 1739268479271518877288942 \\
14 & 590686931539550985679107660268 \\
15 & 846429051478198751690097659025763202 \\
\hline
\end{tabular}

Theorem 5. Let $n, p, q \in \mathbb{N}$. For $1 \leqslant q<p$ denote

$$
\begin{aligned}
K(p, q)=\sum_{j \vdash q-1} \sum_{k \vdash p-q} & {\left[\left(\prod_{i=1}^{q-1} j_{i} ! i^{j_{i}} \prod_{i=1}^{p-q} k_{i} ! i^{k_{i}}\right)^{-1} \prod_{a=1}^{\left\lfloor\frac{n}{2}\right\rfloor}\left(1+\sum_{d \mid a} d j_{d}\right)^{k_{2 a}}\left(1+\sum_{d \mid 2 a} d j_{d}\right)^{a k_{2 a}} .\right.} \\
& \left.\prod_{a=1}^{\left\lfloor\frac{n+1}{2}\right\rfloor}\left(1+\sum_{d \mid 2 a-1} d j_{d}\right)^{a k_{2 a-1}} \prod_{a<b}\left(1+\sum_{d \mid \operatorname{lcm}(a, b)} d j_{d}\right)^{k_{a} k_{b} \operatorname{gcd}(a, b)}\right] .
\end{aligned}
$$

Then the number of nilpotent, commutative semigroups of degree 3 and order $n$ up to isomorphism equals

$$
\sum_{m=2}^{c(n)}(K(n, m)-K(n-1, m-1)) \quad \text { where } c(n)=\lfloor n+3 / 2-\sqrt{2 n+1 / 4}\rfloor .
$$

To determine the number of nilpotent semigroups of degree 3 up to isomorphism or up to isomorphism or anti-isomorphism, we use the technique of power group enumeration in a similar way as Harrison did for universal algebras [Har66]. In Section 5 we present the relevant background material and a number of technical results in preparation for Section 6 where we give the proofs for Theorems 2, 3, and 5 .

\subsection{Bounds and asymptotics}

The formula for the number of nilpotent semigroups of degree 3 up to isomorphism or antiisomorphism in Theorem 3 provides a new lower bound for the number of semigroups up to isomorphism or anti-isomorphism of a given size. Presumably this bound is asymptotic, 
Table 6: Numbers of commutative nilpotent semigroups of degree 3 up to isomorphism

\begin{tabular}{rl}
\hline$n$ & number of non-isomorphic commutative nilpotent semigroups of degree 3 of order $n$ \\
\hline 3 & 1 \\
4 & 5 \\
5 & 23 \\
6 & 155 \\
7 & 2106 \\
8 & 79997 \\
9 & 9350240 \\
10 & 3377274621 \\
11 & 4305807399354 \\
12 & 23951673822318901 \\
13 & 608006617857847433462 \\
14 & 63282042551031180915403659 \\
15 & 25940470166038603666194391357972 \\
16 & 45946454978824286601551283052739171318 \\
17 & 452361442895926947438998019240982893517749169 \\
18 & 30258046596218438115657059107812634405962381166457711 \\
19 & 12094270656160403920767935604624748908993169949317454767617795 \\
\hline
\end{tabular}

that is, the ratio tends to 1 while the order tends to infinity, although this is not a consequence of the result for semigroups up to equality in [KRS76]. The comparison in Table 7 shows also that the lower bound $z(n) / 2 n$ ! from [JMS91] seems to converge rapidly towards our new bound. Analogous observations can be made considering only commutative semigroups though the convergence appears slower as mentioned by Grillet in the analysis in [Gri03].

Our formulae also yield a large qualitative improvement over the old lower bound since they give exact numbers of nilpotent semigroups of degree 3. In particular, the provided numbers can be used to cut down the effort required in an exhaustive search to determine the number of semigroups of a given order, as already done for semigroups of order 9 in [Dis10].

The conjectured asymptotic behaviour of the lower bound of $z(n) / 2 n$ ! for the number of semigroups of order $n$ would imply that almost all sets of isomorphic semigroups on $\{1,2, \ldots, n\}$ are of size $n$ !. In other words, most semigroups have trivial automorphism group; a property that is known for various types of algebraic and combinatorial objects, for example graphs [ER63]. Our formulae could help to prove this conjecture at least for nilpotent semigroups of degree 3 . In each summand in (1) those semigroups of degree 3 are counted for which a bijection with cycle structure corresponding to the partitions $j$ and $k$ is an automorphism. It remains to estimate the contribution of all summands that do not correspond to the identity map. 
Table 7: Numbers of semigroups and nilpotent semigroups of degree 3

\begin{tabular}{rrrr}
\hline$n$ & $\begin{array}{r}\text { number of semigroups } \\
\text { up to isomorphism } \\
\text { or anti-isomorphism }\end{array}$ & $\begin{array}{r}\text { number of semigroups of } \\
\text { degree 3 up to isomorphism } \\
\text { or anti-isomorphism }\end{array}$ & lower bound $\lceil z(n) / 2 n !\rceil$ \\
\hline 3 & 18 & 1 & 1 \\
4 & 126 & 8 & 4 \\
5 & 1160 & 84 & 49 \\
6 & 15973 & 2660 & 2146 \\
7 & 836021 & 609797 & 589691 \\
8 & 1843120128 & 1831687022 & 1827235556 \\
9 & 52989400714478 & 52966239062973 & 52952220887436 \\
10 & unknown & 12417282095522918811 & 12416804146790463082 \\
\hline
\end{tabular}

\section{Construction of nilpotent semigroups of degree 2 or 3}

In this section we describe how to construct nilpotent semigroups of degree 2 or 3 on an $n$-element set. A similar construction is given in [KRS76]. For the sake of brevity we will denote by $[n]$ the set $\{1,2, \ldots, n\}$ where $n \in \mathbb{N}$.

Definition 6. Let $n \geqslant 2$, let $A$ be a non-empty proper subset of $[n]$, and let $B$ denote the complement of $A$ in [n]. If $z \in B$ is arbitrary and $\psi: A \times A \rightarrow B$ is any function, then we can define multiplication on $[n]$ by

$$
x y= \begin{cases}\psi(x, y) & \text { if } x, y \in A \\ z & \text { otherwise. }\end{cases}
$$

We will denote the set $[n]$ with the operation given above by $H(A, \psi, z)$.

Any product $a b c$ in $H(A, \psi, z)$ equals $z$, and so the multiplication defined in (3) is associative. It follows that $H(A, \psi, z)$ is a nilpotent semigroup of degree 2 or 3 . The semigroup $H(A, \psi, z)$ has degree 2 if and only if $H(A, \psi, z)$ is a zero semigroup if and only if $\psi$ is the constant function with value $z$. Conversely, if $T$ is a nilpotent semigroup of degree 3 with elements $[n]$, then setting $A=T \backslash T^{2}$, letting $\psi: A \times A \rightarrow T^{2}$ be defined by $\psi(x, y)=x y$ for all $x, y \in T$, and setting $z$ to be the zero element of $T$, we see that $T=H(A, \psi, z)$. Therefore when enumerating nilpotent semigroups of degree 3 it suffices to consider the semigroups $H(A, \psi, z)$.

\section{Semigroups and commutative semigroups of degree 3 up to equality}

Denote by $Z_{n}$ the set of nilpotent semigroups of degree 3 on $\{1,2, \ldots, n\}$. A formula for the cardinality of a proper subset of $Z_{n}$ is stated in Theorem 15.3 of [JMS91]. However, the 
formula given in [JMS91] actually yields $\left|Z_{n}\right|$ and this is what the proof of the theorem in [JMS91] shows. Similarly, the formula in Theorem 15.8 of [JMS91] can be used to determine the number of all commutative semigroups in $Z_{n}$ even though the statement says otherwise. For the sake of completeness and to avoid confusion we prove that the formulae as given in Theorem 1 are correct.

Proof of Theorem 1. In both parts of the proof, we let $A$ be a fixed non-empty proper subset of $[n]=\{1,2, \ldots, n\}$, let $B$ denote the complement of $A$ in $[n]$, let $m=|B|$, and let $z \in B$ be fixed. We consider semigroups of the form $H(A, \psi, z)$ where $\psi: A \times A \rightarrow B$ as given in Definition 6.

(i). The number of functions from $A \times A$ to $B$ is $m^{(n-m)^{2}}$. To avoid counting semigroups twice for different $m$, we will only consider those functions $\psi$ where every element in $B \backslash\{z\}$ appears in the image of $\psi$. For a subset $X$ of $B \backslash\{z\}$ of size $i$, there are $(m-i)^{(n-m)^{2}}$ functions with no element from $X$ in their image. Using the InclusionExclusion Principle, the number of functions from $A \times A$ to $B$ with image containing $B \backslash\{z\}$ is

$$
\sum_{i=0}^{m-1}(-1)^{i}\left(\begin{array}{c}
m-1 \\
i
\end{array}\right)(m-i)^{(n-m)^{2}} .
$$

The function $\psi$ is defined on a set with $(n-m)^{2}$ elements. Hence the condition that $B \backslash\{z\}$ is contained in the image of $\psi$ implies that $m-1 \leqslant(n-m)^{2}$. Reformulation yields

$$
m \leqslant n+1 / 2-\sqrt{n-3 / 4}
$$

If $m=1$, then every function $\psi: A \times A \rightarrow B$ is constant, and so, as mentioned above, $H(A, \psi, z)$ is not nilpotent of degree 3. Summing (4) over all appropriate values of $m$, the $\left(\begin{array}{l}n \\ m\end{array}\right)$ choices for $B$ and the $m$ choices for $z \in B$ concludes the proof of this part.

(ii). If $H(A, \psi, z)$ is a commutative semigroup, then the function $\psi: A \times A \rightarrow B$ is defined by its values on pairs $(i, j)$ with $i \leqslant j$. There are $(n-m)(n-m+1) / 2$ such pairs and hence there are $m^{(n-m)(n-m+1) / 2}$ such functions $\psi$.

The rest of the proof follows the same steps as the proof of part (i) with $m^{(n-m)(n-m+1) / 2}$ replacing $m^{(n-m)^{2}}$ and where the inequality $m-1 \leqslant(n-m)(n-m+1) / 2$ yields the parameter $c(n)$.

\section{Power group enumeration}

In this section, we shall introduce the required background material relating to power group enumeration and determine the cycle indices of certain power groups necessary to prove our main theorems. The presentation in this section is based on [HP73].

Let $X$ be a non-empty set and let $S_{X}$ denote the symmetric group on $X$. We again denote the set $\{1,2, \ldots, n\}$ by $[n]$, and will write $S_{n}$ instead of $S_{X}$ if $X=[n]$. For a permutation $\pi \in S_{X}$, let $\delta(\pi, k)$ denote the number of cycles of length $k$ in the disjoint cycle decomposition of $\pi$. 
Definition 7. Let $G$ be a subgroup of $S_{n}$. Then the polynomial

$$
\mathcal{Z}\left(G ; x_{1}, x_{2}, \ldots, x_{n}\right)=\frac{1}{|G|} \sum_{g \in G} \prod_{k=1}^{n} x_{k}^{\delta(g, k)}
$$

is called the cycle index of the group $G$; in short, we write $\mathcal{Z}(G)$.

The cycle structure of a permutation $\pi \in S_{n}$ corresponds to a partition of $n$, and all elements with the same cycle structure form a conjugacy class of $S_{n}$. Remember that if $j$ is a partition of $n$, written as $j \vdash n$, then we denote by $j_{i}$ the number of summands equalling $i$. This yields $j_{i}=\delta(\pi, i)$ for all $i$ and for each element $\pi$ in the conjugacy class corresponding to $j$. This observation allows us to write the cycle index of the symmetric group in a compact form.

Lemma 8 ([HP73, (2.2.5)]). The cycle index of $S_{n}$ is

$$
\mathcal{Z}\left(S_{n}\right)=\sum_{j \vdash n}\left(\prod_{i=1}^{n} j_{i} ! i^{j_{i}}\right)^{-1} \prod_{a=1}^{n} x_{a}^{j_{a}} .
$$

In what follows we require actions other than the natural action of the symmetric group $S_{X}$ on $X$. In particular, we require actions on functions in which two groups act independently on the domains and on the images of the functions. If $G$ is a group acting on a set $X$, then we denote by $x^{g}$ the image of $x \in X$ under the action of $g \in G$.

Definition 9. Let $A$ and $B$ be subgroups of $S_{X}$ and $S_{Y}$, respectively, where $X$ and $Y$ are finite disjoint sets. Then we define an action of the group $A \times B$ on the set $Y^{X}$ of functions from $X$ to $Y$ in the following way: the image of $f \in Y^{X}$ under $(\alpha, \beta) \in A \times B$ is given by

$$
f^{(\alpha, \beta)}(x)=\left(f\left(x^{\alpha}\right)\right)^{\beta}
$$

for all $x \in X$. We will refer to $A \times B$ with this action as a power group.

The cycle index itself is not required for the power groups used in this paper. Of interest is the constant form of the Power Group Enumeration Theorem given below, which states the number of orbits under the action of a power group. The result goes back to de Bruijn [dB59], but is presented here in the form given in [HP73, Section 6.1].

Theorem 10. Let $A \times B$ be a power group acting on the functions $Y^{X}$ as in Definition 9. Then the number of orbits of $A \times B$ on $Y^{X}$ equals

$$
\frac{1}{|B|} \sum_{\beta \in B} \mathcal{Z}\left(A ; c_{1}(\beta), c_{2}(\beta), \ldots, c_{|X|}(\beta)\right),
$$

where

$$
c_{i}(\beta)=\sum_{d \mid i} d \delta(\beta, d)
$$


To apply Theorem 10 in the enumeration of nilpotent semigroups of degree 3 we require the cycle indices of the specific group actions defined below.

Definition 11. Let $A$ be a group acting on a set $X$. Then we define:

(i) by $A^{\times 2}$ the group $A$ acting on $X \times X$ componentwise, that is,

$$
\left(x_{1}, x_{2}\right)^{\alpha}=\left(x_{1}^{\alpha}, x_{2}^{\alpha}\right)
$$

for $\alpha \in A$;

(ii) by $2 A^{\times 2}$ the group $S_{2} \times A$ acting on $X \times X$ by

$$
\left(x_{1}, x_{2}\right)^{(\pi, \alpha)}=\left(x_{1^{\pi}}^{\alpha}, x_{2^{\pi}}^{\alpha}\right)
$$

for $\alpha \in A$ and $\pi \in S_{2}$.

(iii) by $A^{\{2\}}$ the group $A$ acting pointwise on the set $\left\{\left\{x_{1}, x_{2}\right\} \mid x_{i} \in X\right\}$ of subsets of a set $X$ with 1 or 2 elements, that is,

$$
\left\{x_{1}, x_{2}\right\}^{\alpha}=\left\{x_{1}^{\alpha}, x_{2}^{\alpha}\right\}
$$

for $\alpha \in A$.

We will show in Section 6 that it is possible to distinguish nilpotent semigroups of degree 3 of the form $H(A, \psi, z)$ as defined in Definition 6 up to isomorphism, and up to isomorphism or anti-isomorphism, by determining the orbit the function $\psi$ belongs to under certain power groups derived from the actions in Definition 11.

In the next lemma, we obtain the cycle indices of the groups $S_{n}^{\times 2}, S_{n}^{\{2\}}$, and $2 S_{n}^{\times 2}$ using the cycle index of $S_{n}$ given in Lemma 8.

Lemma 12. For $n \in \mathbb{N}$ the following hold:

(i) the cycle index of $S_{n}^{\times 2}$ is

$$
\mathcal{Z}\left(S_{n}^{\times 2}\right)=\sum_{j \vdash n}\left(\prod_{i=1}^{n} j_{i} ! i^{j_{i}}\right)^{-1} \prod_{a, b=1}^{n} x_{\operatorname{lcm}(a, b)}^{j_{a} j_{b} \operatorname{gcd}(a, b)} ;
$$

(ii) the cycle index of $2 S_{n}^{\times 2}$ is

$$
\mathcal{Z}\left(2 S_{n}^{\times 2}\right)=\frac{1}{2} \mathcal{Z}\left(S_{n}^{\times 2}\right)+\frac{1}{2} \sum_{j \vdash n}\left(\prod_{i=1}^{n} j_{i} ! i^{j_{i}}\right)^{-1} \prod_{a=1}^{n}\left(q_{a}^{j_{a}} p_{a, a}^{j_{a}^{2}-j_{a}} \prod_{b=1}^{a-1} p_{a, b}^{2 j_{a} j_{b}}\right),
$$

where $p_{a, b}=x_{\operatorname{lcm}(2, a, b)}^{a b / \operatorname{lcm}(2, a, b)}$ and

$$
q_{a}=\left\{\begin{array}{lll}
x_{a} x_{2 a}^{(a-1) / 2} & \text { if } a \equiv 1 & \bmod 2 \\
x_{a}^{a} & \text { if } a \equiv 0 & \bmod 4 \\
x_{a / 2}^{2} x_{a}^{a-1} & \text { if } a \equiv 2 & \bmod 4
\end{array}\right.
$$


(iii) the cycle index of $S_{n}^{\{2\}}$ is

$$
\mathcal{Z}\left(S_{n}^{\{2\}}\right)=\sum_{j \vdash n}\left(\prod_{i=1}^{n} j_{i} ! i^{j_{i}}\right)^{-1} \prod_{a=1}^{\lfloor n / 2\rfloor} r_{a} \prod_{a=1}^{\lfloor(n+1) / 2\rfloor} s_{a} \prod_{a=1}^{n} t_{a}\left(\prod_{b=1}^{a-1} x_{\operatorname{lcm}(a, b)}^{j_{a} j_{b} \operatorname{gcd}(a, b)}\right)
$$

where the monomials are $r_{a}=x_{a}^{j_{2 a}} x_{2 a}^{a j_{2 a}}, s_{a}=x_{2 a-1}^{a j_{2 a-1}}$, and $t_{a}=x_{a}^{a\left(j_{a}^{2}-j_{a}\right) / 2}$.

Proof. (i). By definition each permutation in $S_{n}$ induces a permutation in $S_{n}^{\times 2}$. Let $\alpha \in S_{n}$ and let $z_{a}$ and $z_{b}$ be two cycles thereof with length $a$ and $b$ respectively. Consider the action of $\alpha$ on those pairs in $[n] \times[n]$ which have as first component an element in $z_{a}$ and as second component an element in $z_{b}$. Let $(i, j) \in[n] \times[n]$ be one such pair. Since $i^{\alpha^{k}}=i$ if and only if $a$ divides $k$, and $j^{\alpha^{k}}=j$ if and only if $b$ divides $k$, the pair $(i, j)$ is in an orbit of length $\operatorname{lcm}(a, b)$. The total number of pairs with first component in $z_{a}$ and second component in $z_{b}$ equals $a b$. Hence the number of orbits equals $\operatorname{gcd}(a, b)$. Repeating this consideration for every pair of cycles in $\alpha$ leads to

$$
\prod_{a, b=1}^{n} x_{\operatorname{lcm}(a, b)}^{\delta(\alpha, a) \delta(\alpha, b) \operatorname{gcd}(a, b)}
$$

as the summand corresponding to $\alpha$ in the cycle index $\mathcal{Z}\left(S_{n}^{\times 2}\right)$. This yields

$$
\mathcal{Z}\left(S_{n}^{\times 2}\right)=\frac{1}{n !} \sum_{\alpha \in S_{n}} \prod_{a, b=1}^{n} x_{\operatorname{lcm}(a, b)}^{\delta(\alpha, a) \delta(\alpha, b) \operatorname{gcd}(a, b)} .
$$

That the contribution of $\alpha$ to $\mathcal{Z}\left(S_{n}^{\times 2}\right)$ only depends on its cycle structure allows us to replace the summation over all group elements by a summation over partitions of $n$; one for each conjugacy class of $S_{n}$. The number of elements with cycle structure associated to a partition $j \vdash n$ equals $n ! / \prod_{i=1}^{n} j_{i} ! i^{j_{i}}$. Therefore

$$
\mathcal{Z}\left(S_{n}^{\times 2}\right)=\frac{1}{n !} \sum_{j \vdash n} \frac{n !}{\prod_{i=1}^{n} j_{i} ! i^{j_{i}}} \prod_{a, b=1}^{n} x_{\operatorname{lcm}(a, b)}^{j_{a j} j_{b} \operatorname{gcd}(a, b)},
$$

and cancelling the factor $n$ ! concludes the proof.

(ii). For elements $\left(\operatorname{id}_{\{1,2\}}, \alpha\right) \in 2 S_{n}^{\times 2}$ the contribution to the cycle index of $2 S_{n}^{\times 2}$ equals the contribute of $\alpha$ to $\mathcal{Z}\left(S_{n}^{\times 2}\right)$ given in (i). It is rearranged as follows to illustrate which contributions come from identical cycles and which from disjoint cycles:

$$
\prod_{a, b=1}^{n} x_{\operatorname{lcm}(a, b)}^{\delta(\alpha, a) \delta(\alpha, b) \operatorname{gcd}(a, b)}=\prod_{a=1}^{n}\left(x_{a}^{a \delta(\alpha, a)} x_{a}^{a\left(\delta(\alpha, a)^{2}-\delta(\alpha, a)\right)} \prod_{b<a} x_{\operatorname{lcm}(a, b)}^{2 \delta(\alpha, a) \delta(\alpha, b) \operatorname{gcd}(a, b)}\right) .
$$

For group elements of the form $((12), \alpha)$ the contribution is going to be deduced from the one of $\alpha$. Let $z_{a}$ and $z_{b}$ again be two cycles in $\alpha$ of length $a$ and $b$ respectively, and assume at first, they are disjoint. Then $z_{a}$ and $z_{b}$ induce $2 \operatorname{gcd}(a, b)$ orbits of length 
$\operatorname{lcm}(a, b)$ on the $2 a b$ pairs in $[n] \times[n]$ with one component from each of the two cycles. Let

$$
\omega=\left\{\left(i_{1}, j_{1}\right),\left(i_{2}, j_{2}\right), \ldots,\left(i_{\operatorname{lcm}(a, b)}, j_{\operatorname{lcm}(a, b)}\right)\right\}
$$

be such an orbit. Then

$$
\bar{\omega}=\left\{\left(j_{1}, i_{1}\right),\left(j_{2}, i_{2}\right), \ldots,\left(j_{\operatorname{lcm}(a, b)}, i_{\operatorname{lcm}(a, b)}\right)\right\}
$$

is another one. The set $\omega \cup \bar{\omega}$ is closed under the action of $((12), \alpha)$. In how many orbits $\omega \cup \bar{\omega}$ splits depends on the parity of $a$ and $b$. Acting with $((12), \alpha)$ on $\left(i_{1}, j_{1}\right)$ for $\operatorname{lcm}(a, b)$ times gives $\left(i_{1}, j_{1}\right)$ if $\operatorname{lcm}(a, b)$ is even and $\left(j_{1}, i_{1}\right)$ if $\operatorname{lcm}(a, b)$ is odd. Hence the two orbits $\omega$ and $\bar{\omega}$ merge to one orbit in the latter case and give two new orbits of the original length otherwise. This yields the monomial

$$
x_{\operatorname{lcm}(2, a, b)}^{2 a b / \operatorname{lcm}(2, a, b)}=\left\{\begin{array}{lll}
x_{\operatorname{lcm}(a, b)}^{2 \operatorname{gcd}(a, b)} & \text { if } & \operatorname{lcm}(a, b) \equiv 0 \bmod 2 \\
x_{2 \operatorname{lcm}(a, b)}^{\operatorname{gcd}(a, b)} & \text { if } & \operatorname{lcm}(a, b) \equiv 1 \bmod 2,
\end{array}\right.
$$

which appears $\delta(\alpha, a) \delta(\alpha, b)$ times if $a \neq b$ and $\left(\delta(\alpha, a)^{2}-\delta(\alpha, a)\right) / 2$ times if $a=b$.

Let $z_{a}$ and $z_{b}$ now be identical and equal to the cycle $\left(i_{1} i_{2} \cdots i_{a}\right)$. The contribution to the monomial of $\alpha$ is the factor $x_{a}^{a}$. The orbits are of the form

$$
\left\{\left(i_{g}, i_{h}\right) \mid 1 \leqslant g, h \leqslant a, g \equiv h+s \bmod a\right\}
$$

for $0 \leqslant s \leqslant a-1$. For $s=0$ the orbit consists of pairs with equal entries, that is, $\left\{\left(i_{1}, i_{1}\right),\left(i_{2}, i_{2}\right) \ldots\left(i_{a}, i_{a}\right)\right\}$, and thus stays the same under $((12), \alpha)$. For an orbit $\omega=$ $\left\{\left(i_{g}, i_{h}\right) \mid 1 \leqslant g, h \leqslant a, g \equiv h+s \bmod a\right\}$ with $s \neq 0$ define $\bar{\omega}$ as in $(7)$. If $\omega \neq \bar{\omega}$ one argues like in the case of two disjoint cycles and gets the result depending on the parity of $a$. Note that $\omega=\bar{\omega}$ if and only if $s=a / 2$. In particular this does not occur for $a$ odd in which case

$$
x_{a} x_{2 a}^{(a-1) / 2}
$$

is the factor contributed to the monomial of $((12), \alpha)$. If on the other hand $a$ is even, one more case split is needed to deal with the orbit

$$
\omega=\left\{\left(i_{g}, i_{h}\right) \mid 1 \leqslant g, h \leqslant a, g \equiv h+a / 2 \bmod a\right\} .
$$

Acting with $((12), \alpha)$ on $\left(i_{a}, i_{a / 2}\right)$ for $a / 2$ times gives $\left(i_{a}, i_{a / 2}\right)$ if $a / 2$ is odd and $\left(i_{a / 2}, i_{a}\right)$ if $a / 2$ is even. Thus $\omega$ splits into two orbits of length $a / 2$ in the former case and stays one orbit in the latter. The resulting factors contributed to the monomial of $((12), \alpha)$ are therefore

$$
\begin{array}{rlll}
x_{a}^{a} & \text { if } & a \equiv 0 & \bmod 4 \\
x_{a / 2}^{2} x_{a}^{a-1} & \text { if } & a \equiv 2 & \bmod 4 .
\end{array}
$$

Following the analysis for all pairs of cycles in $\alpha$ leads to the contribution of $((12), \alpha)$ to the cycle index. Summing as before over all partitions of $n$, which correspond to the different cycle structures, proves the formula for $\mathcal{Z}\left(2 S_{n}^{\times 2}\right)$. 
(iii). To compute $\mathcal{Z}\left(S_{n}^{\{2\}}\right)$ let $\omega$ and $\bar{\omega}$ as in (6) and (7) be orbits for two cycles $z_{a}$ and $z_{b}$ from $\alpha \in S_{n}$ acting on $[n] \times[n]$. If the two cycles $z_{a}$ and $z_{b}$ are disjoint then both $\omega$ and $\bar{\omega}$ correspond to the same orbit

$$
\left\{\left\{i_{1}, j_{1}\right\},\left\{i_{2}, j_{2}\right\}, \ldots,\left\{i_{\mathrm{lcm}(a, b)}, j_{\mathrm{lcm}(a, b)}\right\}\right\}
$$

of $\alpha$ acting on $[n]^{\{2\}}$. The contribution to the monomial of $\alpha$ in $\mathcal{Z}\left(S_{n}^{\{2\}}\right)$ is therefore $x_{\operatorname{lcm}(a, b)}^{\operatorname{gcd}(a, b)}$. Let $z_{a}$ and $z_{b}$ now be identical and equal to the cycle $\left(i_{1} i_{2} \cdots i_{a}\right)$. In $S_{n}^{\times 2}$ this gave rise to the orbits $\left\{\left(i_{g}, i_{h}\right) \mid 1 \leqslant g, h \leqslant a, g \equiv h+s \bmod a\right\}$ for $0 \leqslant s \leqslant a-1$. The corresponding orbit under $S_{n}^{\{2\}}$ for $s=0$ becomes $\left\{\left\{i_{1}\right\},\left\{i_{2}\right\}, \ldots,\left\{i_{a}\right\}\right\}$. All other orbits become $\left\{\left\{i_{g}, i_{h}\right\} \mid 1 \leqslant g, h \leqslant a, g \equiv h+s \bmod a\right\}$ in the same way as before, but these are identical for $s$ and $a-s$. This yields one further exception if $a$ is even and $s=a / 2$, in which case the orbit collapses to $\left\{\left\{i_{g}, i_{g+a / 2}\right\} \mid 1 \leqslant g \leqslant a / 2\right\}$. In total, identical cycles lead to the monomials

$$
\begin{array}{cccc}
x_{a / 2} x_{a}^{a / 2} & \text { if } & a \equiv 0 & \bmod 2 \\
x_{a}^{(a+1) / 2} & \text { if } & a \equiv 1 & \bmod 2 .
\end{array}
$$

Summing once more over conjugacy classes and making the case split depending on the parity proves the formula for $\mathcal{Z}\left(S_{n}^{\{2\}}\right)$.

Formulae like those in the previous lemma for slightly different actions are given in $[H P 73,(4.1 .9)]$ and $[H P 73,(5.1 .5)]$. The proof techniques used here are essentially the same as in [HP73].

\section{Proofs of the main theorems}

In this section, we prove Theorem 2. The proofs of Theorems 3 , and 5 are very similar to the proof of Theorem 2, and so, for the sake of brevity we show how to obtain these proofs from the one presented, rather than giving the proofs in detail.

We consider the following sets of nilpotent semigroups of degree 3 : for $m, n \in \mathbb{N}$ with $2 \leqslant m \leqslant n-1$ we define

$$
Z_{n, m}=\{H([n] \backslash[m], \psi, 1) \mid \psi:[n] \backslash[m] \times[n] \backslash[m] \rightarrow[m] \text { with }[m] \backslash\{1\} \subseteq \operatorname{im}(\psi)\},
$$

where $H([n] \backslash[m], \psi, 1)$ is as in Definition 6 , and $[n]$ is short for $\{1,2, \ldots, n\}$, as before. From this point on, we will only consider semigroups belonging to $Z_{n, m}$, and so we write $H(\psi)$ instead of $H([n] \backslash[m], \psi, 1)$.

If $H(\psi) \in Z_{n, m}$ is commutative, then we define a function $\psi^{\prime}$ from the set of subsets of $[n]$ with 1 or 2 elements to $[m]$ by

$$
\psi^{\prime}\{i, j\}=\psi(i, j)
$$

for $i \leqslant j$. Since the equality $\psi(i, j)=\psi(j, i)$ holds for all $i, j$, the function $\psi^{\prime}$ is welldefined. Moreover, every function from the set of subsets of $[n]$ with 1 or 2 elements to $[m]$ is induced in this way by a function $\psi$ such that $H(\psi) \in Z_{n, m}$ and $H(\psi)$ is commutative. 
Lemma 13. Let $S$ be a nilpotent semigroup of degree 3 with $n$ elements. Then $S$ is isomorphic to a semigroup in $Z_{n, m}$ if and only if $m=\left|S^{2}\right|$.

Proof. Let $z$ denote the zero element of $S$, and let $f: S \rightarrow[n]$ be any bijection such that $f(z)=1$ and $f\left(S^{2}\right)=[m]$. Then define $\psi:([n] \backslash[m]) \times([n] \backslash[m]) \rightarrow[m]$ by

$$
\psi(i, j)=f\left(f^{-1}(i) f^{-1}(j)\right) .
$$

Now, since $S$ is nilpotent, if $x \in[m] \backslash\{1\}$, there exist $s, t \in S \backslash S^{2}$ such that $f(s t)=x$. Thus $\psi(f(s), f(t))=x$ and $[m] \backslash\{1\} \subseteq \operatorname{im}(\psi)$. Hence $H(\psi) \in Z_{n, m}$ and it remains to show that $f$ is an isomorphism. If $x, y \in S \backslash S^{2}$, then $f(x) f(y)=\psi(f(x), f(y))=f(x y)$. Otherwise, $x \in S^{2}$ or $y \in S^{2}$, in which case $f(x) f(y)=1=f(z)=f(x y)$.

It follows from Lemma 13 that we can determine the number of isomorphism types in each of the sets $Z_{n, m}$ independently. Of course, if $S$ is a nilpotent semigroup of degree 3 and $m=\left|S^{2}\right|$, then it is not true in general that there exists a unique semigroup in $Z_{n, m}$ isomorphic to $S$. Instead isomorphisms between semigroups in $Z_{n, m}$ induce an equivalence relation on the functions $\psi$, which define the semigroups in $Z_{n, m}$.

If $H(\psi) \in Z_{n, m}$ and $T$ is a nilpotent semigroup of degree 3 such that $H(\psi) \cong T$, then there exists $\pi \in S_{n}$ such that $S^{\pi}=T$. Hence $T \in Z_{n, m}$ if and only if $\pi$ stabilises $[n] \backslash[m]$ and $\{1\}$ - and hence $[m] \backslash\{1\}$ - setwise. In particular, the action of $\pi$ on the domain and range of $\psi$ are independent, and so equivalence can be captured using a power group action.

Lemma 14. For $m, n \in \mathbb{N}$ with $2 \leqslant m \leqslant n-1$ let $H(\psi), H(\chi) \in Z_{n, m}$, and let $U_{m}$ denote the pointwise stabiliser of 1 in $S_{m}$. Then the following hold:

(i) the semigroups $H(\psi)$ and $H(\chi)$ are isomorphic if and only if $\psi$ and $\chi$ are in the same orbit under the power group $S_{[n] \backslash[m]}^{\times 2} \times U_{m}$;

(ii) the semigroups $H(\psi)$ and $H(\chi)$ are isomorphic or anti-isomorphic if and only if $\psi$ and $\chi$ are in the same orbit under the power group $2 S_{[n] \backslash[m]}^{\times 2} \times U_{m}$.

If in addition $H(\psi)$ and $H(\chi)$ are commutative, then:

(iii) the semigroups $H(\psi)$ and $H(\chi)$ are isomorphic if and only if $\psi^{\prime}$ and $\chi^{\prime}$ (as defined in (8)) are in the same orbit under the power group $S_{[n] \backslash[m]}^{\{2\}} \times U_{m}$.

Proof. (i). $(\Rightarrow)$ By assumption there exists $\pi \in S_{n}$ such that $\pi: H(\psi) \rightarrow H(\chi)$ is an isomorphism. From the comments before the lemma, $\pi$ stabilises $[n] \backslash[m]$ and 1 , and so there exist $\tau \in U_{m}$ and $\sigma \in S_{[n] \backslash[m]}$ such that $\tau \sigma=\pi$. Then for all $x, y \in[n] \backslash[m]$

$$
\psi(x, y)=\left(\psi(x, y)^{\pi}\right)^{\pi^{-1}}=\left(\chi\left(x^{\pi}, y^{\pi}\right)\right)^{\pi^{-1}}=\left(\chi\left(x^{\sigma}, y^{\sigma}\right)\right)^{\tau^{-1}} .
$$

It follows that $\chi$ acted on by $\left(\sigma, \tau^{-1}\right) \in S_{[n] \backslash[m]}^{\times 2} \times U_{m}$ equals $\psi$, as required.

$(\Leftarrow)$ As $\psi$ and $\chi$ lie in the same orbit under the action of the power group $S_{[n] \backslash[m]}^{\times 2} \times U_{m}$, there exist $\sigma \in S_{[n] \backslash[m]}$ and $\tau \in U_{m}$ such that $\psi^{(\sigma, \tau)}=\chi$. Let $\pi=\sigma \tau^{-1} \in S_{n}$. We will 
show that $\pi$ is an isomorphism from $H(\psi)$ to $H(\chi)$. Let $x, y \in[n]$ be arbitrary. If $x, y \in[n] \backslash[m]$, then

$$
x^{\pi} y^{\pi}=\psi\left(x^{\sigma}, y^{\sigma}\right)=\left(\psi\left(x^{\sigma}, y^{\sigma}\right)^{\tau}\right)^{\tau^{-1}}=\left(\psi^{(\sigma, \tau)}(x, y)\right)^{\tau^{-1}}=(\chi(x, y))^{\tau^{-1}}=(x y)^{\pi} .
$$

If $x \in[n] \backslash[m]$ and $y \in[m]$, then $(x y)^{\pi}=1^{\pi}=1=x^{\sigma} y^{\tau^{-1}}=x^{\pi} y^{\pi}$. The case when $x \in[m]$ and $y \in[n] \backslash[m]$ and the case when $x, y \in[m]$ follow by similar arguments.

(ii). In this part of the proof we write $(\alpha, \beta, \gamma)$ instead of $((\alpha, \beta), \gamma)$ when referring to elements of $2 S_{[n] \backslash[m]}^{\times 2} \times U_{m}$.

$(\Rightarrow)$ If $H(\psi)$ and $H(\chi)$ are isomorphic, then, by part (i), the functions $\psi$ and $\chi$ are in the same orbit under the action of $S_{[n] \backslash[m]}^{\times 2} \times U_{m}$. Since $S_{[n] \backslash[m]}^{\times 2} \times U_{m}$ is contained in $2 S_{[n] \backslash[m]}^{\times 2} \times U_{m}$, it follows that $\psi$ and $\chi$ are in the same orbit under the action of $2 S_{[n] \backslash[m]}^{\times 2} \times U_{m}$.

If $H(\psi)$ and $H(\chi)$ are not isomorphic, then there exists $\pi \in S_{n}$ such that $\pi: H(\psi) \rightarrow$ $H(\chi)$ is an anti-isomorphism. As in the proof of part (i), there exist $\tau \in U_{m}$ and $\sigma \in$ $S_{[n] \backslash[m]}$ such that $\pi=\tau \sigma$. Then, for all $x, y \in[n] \backslash[m]$,

$$
\psi(x, y)=\left(\psi(x, y)^{\pi}\right)^{\pi^{-1}}=\left(\chi\left(y^{\pi}, x^{\pi}\right)\right)^{\pi^{-1}}=\left(\chi\left(y^{\sigma}, x^{\sigma}\right)\right)^{\tau^{-1}}=\chi^{\left(\sigma, \tau^{-1}\right.}(y, x) .
$$

Hence $\chi$ acted on by $\left((12), \sigma, \tau^{-1}\right) \in 2 S_{[n] \backslash[m]}^{\times 2} \times U_{m}$ equals $\psi$.

$(\Leftarrow)$ If $\psi=\chi^{\left(\mathrm{id}_{\{1,2\}}, \sigma, \tau\right)}$ for some $\left(\operatorname{id}_{\{1,2\}}, \sigma, \tau\right) \in 2 S_{[n] \backslash[m]}^{\times 2} \times U_{m}$, then $H(\psi)$ and $H(\chi)$ are isomorphic by part (i). So, we may assume that $\psi=\chi^{((12), \sigma, \tau)}$. Let $\pi=\sigma \tau^{-1} \in S_{n}$. We show that $\pi$ is an anti-isomorphism from $H(\psi)$ to $H(\chi)$. Let $x, y \in[n]$ be arbitrary. If $x, y \in[n] \backslash[m]$, then

$$
x^{\pi} y^{\pi}=\psi\left(x^{\sigma}, y^{\sigma}\right)=\left(\psi\left(x^{\sigma}, y^{\sigma}\right)^{\tau}\right)^{\tau^{-1}}=\left(\psi^{((12), \sigma, \tau)}(y, x)\right)^{\tau^{-1}}=(\chi(y, x))^{\tau^{-1}}=(y x)^{\pi} .
$$

If $x \in[n] \backslash[m]$ and $y \in[m]$, then $(x y)^{\pi}=1^{\pi}=1=y^{\tau^{-1}} x^{\sigma}=y^{\pi} x^{\pi}$. The case when $x \in[m]$ and $y \in[n] \backslash[m]$ and the case when $x, y \in[m]$ follow by similar arguments.

(iii). The proof follows from (i) and the observation that $\psi^{\prime}$ and $\chi^{\prime}$ are in the same orbit under $S_{[n] \backslash[m]}^{\{2\}} \times U_{m}$ if and only if $\psi$ and $\chi$ are in the same orbit under $S_{[n] \backslash[m]}^{\times 2} \times U_{m}$.

Lemma 14(i) shows that the number of non-isomorphic semigroups in $Z_{n, m}$ equals the number of orbits of functions defining semigroups in $Z_{n, m}$ under the appropriate power group action. Together with Theorem 10 this provides the essential information required to prove the formula given in Theorem 2 for the number of nilpotent semigroups of degree 3 of order $n$ up to isomorphism.

Proof of Theorem 2. Denote by $U_{q}$ the stabiliser of 1 in $S_{q}$. We shall first show that $N(p, q)$ is the number of orbits of the power group $S_{[p] \backslash[q]}^{\times 2} \times U_{q}$ on functions from $([p] \backslash$ $[q]) \times([p] \backslash[q])$ to $[q]$. By Theorem 10 the latter equals

$$
\frac{1}{(q-1) !} \sum_{\beta \in H} \mathcal{Z}\left(S_{[p] \backslash[q]}^{\times 2} ; c_{1}(\beta), \ldots, c_{(p-q)^{2}}(\beta)\right),
$$


where

$$
c_{i}(\beta)=\sum_{d \mid i} d \delta(\beta, d)
$$

If $\beta \in U_{q}$, then $\mathcal{Z}\left(S_{[p] \backslash \backslash q]}^{\times 2} ; c_{1}(\beta), \ldots, c_{(p-q)}(\beta)\right)$ only depends on the cycle structure of $\beta$ and is therefore an invariant of the conjugacy classes of $U_{q}$. These conjugacy classes are in 1-1 correspondence with the partitions of $q-1$. If $j$ is a partition of $q-1$ corresponding to the conjugacy class of $\beta$, then $\delta(\beta, 1)=j_{1}+1$ and $\delta(\beta, i)=j_{i}$ for $i=2, \ldots, q-1$ (where $j_{i}$ denotes, as before, the number of summands in $j$ equalling $i$ ). This yields that $c_{i}(\beta)=1+\sum_{d \mid i} d j_{d}$. The size of the conjugacy class in $U_{q}$ corresponding to the partition $j$ is $(q-1) ! / \prod_{i=1}^{q-1} j_{i} ! i^{j_{i}}$. Hence summing over conjugacy classes in (9) gives:

$$
\sum_{j \vdash q-1}\left(\prod_{i=1}^{q-1} j_{i} ! i^{j_{i}}\right)^{-1} \mathcal{Z}\left(S_{[p] \backslash[q]}^{\times 2} ; 1+\sum_{d \mid 1} d j_{d}, \ldots, 1+\sum_{d \mid(p-q)^{2}} d j_{d}\right) .
$$

Substituting the cycle index of $S_{[p] \backslash[q]}^{\times 2}$ from Lemma 12(i) into (10) yields the formula given in the statement of the Theorem for $N(p, q)$.

By Lemma 14(i), the number of non-isomorphic semigroups in $Z_{n, m}$ for $m \in \mathbb{N}$ with $2 \leqslant m \leqslant n-1$ equals the number of orbits under the power group $S_{[n] \backslash[m]}^{\times 2} \times U_{m}$ of functions from $([n] \backslash[m]) \times([n] \backslash[m])$ to $[m]$ having $[m] \backslash\{1\}$ in their image. The orbits counted in $N(n, m)$ include those of functions which do not contain $[m] \backslash\{1\}$ in their image. The number of such orbits equals $N(n-1, m-1)$, the number of orbits of functions with one fewer element in the image set. Hence the number of non-isomorphic semigroups in $Z_{n, m}$ equals $N(n, m)-N(n-1, m-1)$. With Lemma 13, it follows that the number of non-isomorphic nilpotent semigroups of degree 3 with $n$ elements is

$$
\sum_{m=2}^{a(n)}(N(m, n)-N(m-1, n-1)) \quad \text { where } a(n)=\lfloor n+1 / 2-\sqrt{n-3 / 4}\rfloor .
$$

Replacing the cycle index in (9) by that of $2 S_{[p] \backslash[q]}^{\times 2}$ and $S_{[p] \backslash[q]}^{\{2\}}$ proves Theorems 3 and 5 , respectively, using the same argument as above.

\section{References}

[dB59] N. G. de Bruijn. Generalization of Polya's fundamental theorem in enumerative combinatorial analysis. Nederl. Akad. Wetensch. Proc. Ser. A 62 = Indag. Math., 21:59-69, 1959.

[Dis10] Andreas Distler. Classification and Enumeration of Finite Semigroups. Shaker Verlag, Aachen, 2010. also PhD thesis, University of St Andrews, 2010, http: //hdl.handle.net/10023/945.

[DK09] Andreas Distler and Tom Kelsey. The monoids of orders eight, nine \& ten. Ann. Math. Artif. Intell., 56(1):3-21, 2009. 
[DM11] A. Distler and J. D. Mitchell. Smallsemi - a GAP package, version 0.6.4, 2011. http://tinyurl.com/jdmitchell/smallsemi/.

[ER63] P. Erdős and A. Rényi. Asymmetric graphs. Acta Math. Acad. Sci. Hungar, 14:295-315, 1963.

[GAP08] The GAP Group. GAP - Groups, Algorithms, and Programming, Version 4.4.12, 2008. http://www.gap-system.org.

[Gri03] Pierre Antoine Grillet. Computing finite commutative semigroups. II, III. Semigroup Forum, 67(2):159-184, 185-204, 2003.

[Har66] Michael A. Harrison. The number of isomorphism types of finite algebras. Proc. Amer. Math. Soc., 17:731-737, 1966.

[HP73] Frank Harary and Edgar M. Palmer. Graphical enumeration. Academic Press, New York, 1973.

[JMS91] H. Jürgensen, F. Migliorini, and J. Szép. Semigroups. Akadémiai Kiadó (Publishing House of the Hungarian Academy of Sciences), Budapest, 1991.

[KRS76] Daniel J. Kleitman, Bruce R. Rothschild, and Joel H. Spencer. The number of semigroups of order n. Proc. Amer. Math. Soc., 55(1):227-232, 1976.

[Pol37] G. Polya. Kombinatorische Anzahlbestimmungen für Gruppen, Graphen und chemische Verbindungen. Acta Math., 68(1):145-254, 1937.

[Red27] J. Howard Redfield. The Theory of Group-Reduced Distributions. Amer. J. Math., 49(3):433-455, 1927.

[Sla95] John Slaney. FINDER - Finite Domain Enumerator, Version 3.0, 1995. ftp: //arp.anu.edu.au/pub/papers/slaney/finder/finder.ps.gz.

[SYT94] S. Satoh, K. Yama, and M. Tokizawa. Semigroups of order 8. Semigroup Forum, 49(1):7-29, 1994. 